\title{
Sillages tourbillonnaires des structures immergées
}

\section{Adrien POUPARDIN ${ }^{1}$, Nicolas BOURNETON ${ }^{1}$, Gaële PERRET ${ }^{1}$, Grégory PINON ${ }^{1}$, Elie RIVOALEN ${ }^{2}$, Jérôme BROSSARD ${ }^{1}$}

1. Laboratoire Ondes et Milieux Complexes, FRE/CNRS 3102 et Université du Havre, Bâtiment COREVA, 53 rue de Prony, BP 540, 76058 Le Havre Cedex, France. adrien.poupardin@etu.univ-lehavre.fr ; Nicolas.Bourneton@developpement-durable.gouv.fr

2. Laboratoire de Mécanique de Rouen, EA 3828, INSA de Rouen, Avenue de l'Université, BP 08, 76801 Saint Etienne du Rouvray, France.

\section{Résumé :}

Les structures immergées sous le niveau de la mer, pour atténuer ou récupérer l'énergie de la houle, sont soumises à l'action des vagues et engendrent le développement d'un sillage tourbillonnaire plus ou moins complexe en fonction de la géométrie de la structure. Ces perturbations posent à la fois des problèmes de dimensionnement des structures et d'environnement. En effet, les tourbillons sont les signes d'efforts de portance qui peuvent être dimensionnants pour la structure. Par ailleurs, la modification des conditions hydrauliques peut provoquer des phénomènes d'affouillement au voisinage de l'ouvrage. Afin de comprendre, de quantifier et de modéliser, les phénomènes associés aux écoulements des ouvrages immergés, nous traiterons un problème géométriquement simple comme celui d'une plaque horizontale immergée de faible épaisseur.

Nous décrirons et qualifierons le sillage tourbillonnaire (2D) d'une plaque mince soumise à une houle incidente: naissance des tourbillons principaux, trajectoires et évolutions de ces structures tourbillonnaires. Les résultats expérimentaux et numériques seront confrontés pour décrire la dynamique tourbillonnaire associée au sillage de la plaque.

Mots-clés :

Dynamique tourbillonnaire- Structure immergée - Sillage - Impacts environnementaux

\begin{abstract}
:
The interaction of wave fields with energy recovery systems produces the development of boundary layers and wake vortices which depend on the geometry of submerged structures. These disturbances lead to problems in terms of structures design and in terms of environmental impact. In fact, the presence of vortices is indicative of lift forces which may influence the structure design; the perturbed local hydrodynamics may generate scouring processes. In order to understand, quantify and model the environmental impact generated by submerged structures, an experiment has been
\end{abstract}


developed some years ago which used a horizontal thin plate structure to simplify the problem (BOULIER, 1996; BOULIER et al., 1997). Recent tools of velocity field measurement and visualization (PIV), with a good spatial resolution, enable to follow the dynamics of vortices generated by submerged structures. A lagrangian simulation (using vortex method) of the experiment enables to reproduce numerically the vortex wake in non stationary, two-dimensional and incompressible flows.

We will describe and quantify the vortex wake (2D) of a horizontal thin plate in interaction with a wave field: formation of main vortex, trajectories and evolutions of these structures. The experimental and numerical results will be presented to describe the vortex dynamics close to the thin plate.

Key words:

Vortex dynamics - Submerged structure - Wake - Environmental impacts

\section{Introduction}

Les structures immergées de type plaque horizontale ont la propriété de réfléchir une partie de l'énergie de la houle, d'en dissiper une seconde, et ainsi de protéger les côtes (PATARAPANICH \& CHEONG, 1989). Elles peuvent également être utilisées comme convertisseur d'énergie en plaçant des hydro-turbines immédiatement au dessous d'elles (GRAW, 1993).

Ces plaques immergées posent des problèmes d'affouillement des fonds marins. En effet, des tourbillons sont générés en bout de plaque et sont advectés vers le fond. Des études d'affouillement ont principalement été menées dans le cas de marches ou de digues immergées (SUMER et al., 2001) car l'équilibre des structures peut être remis en cause par ces phénomènes.

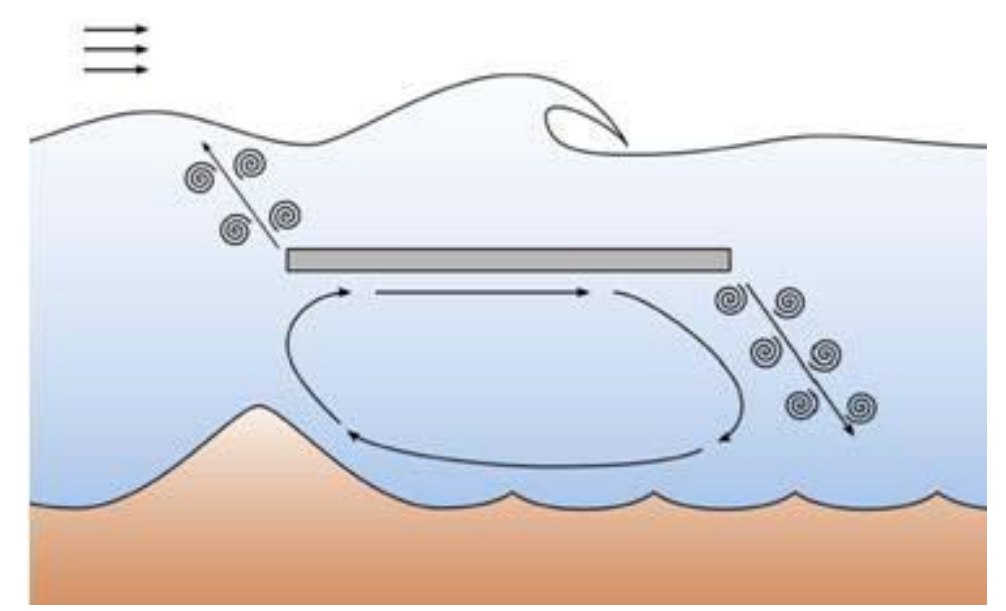

Figure 1. Représentation schématique d'un écoulement oscillant autour d'une plaque immergée (illustration: CHERFILS et al., 2009). 
Les théories potentielles (PATARAPANICH, 1984 ; MASSEL, 1983 ; CARTER, 2005) sont souvent suffisantes pour décrire la déformation de la surface libre et calculer les coefficients de réflexion et de transmission. Mais, leur incapacité à reproduire la génération de tourbillons conduisent à mettre en œuvre des méthodes de résolution numériques de l'équation de Navier-Stokes (CHANG et al., 2001 ; CHANG et al., 2005 ; HUANG \& DONG, 1999 ; TING \& KIM, 1994). Les résultats obtenus montrent, par exemple, que le dessous d'une plaque immergée n'est pas le meilleur endroit pour récupérer de l'énergie comme le propose Graw.

La présence d'une surface libre déformable conduit à des difficultés de résolution pour les méthodes numériques avec maillage. Une modélisation lagrangienne (vortex method), sans maillage, de l'écoulement permet de reproduire numériquement le sillage tourbillonnaire dans le cadre des écoulements instationnaires, bidimensionnels et incompressibles (BOULIER, 1996).

$\mathrm{Du}$ point de vue expérimental, les outils récents, à haute résolution spatiale, de visualisation et de mesure de champs de vitesse (PIV) permettent de nouvelles investigations et ainsi de poursuivre l'étude de BOULIER (1996).

\section{Etudes expérimentales}

Pour caractériser les tourbillons générés en bout de plaque nous avons installé une plaque mince horizontale de dimensions $295 \times 250 \times 2 \mathrm{~mm}$ dans un canal à houle d'une longueur de $10 \mathrm{~m}$ et d'une largeur de $30 \mathrm{~cm}$. Pour notre étude nous avons utilisé une hauteur d'eau de $20 \mathrm{~cm}$ et une immersion de la plaque de $6,8 \mathrm{~cm}$. La houle monochromatique, générée par un batteur oscillant, a une fréquence de $1 \mathrm{~Hz}$ et une amplitude de $11 \pm 1 \mathrm{~mm}$. A l'autre extrémité du canal une plage d'amortissement permet d'atténuer fortement la réflexion de la houle ( $\mathrm{Cr} \sim 5 \%)$.

Cette configuration expérimentale a été choisie car elle conduit à une assez faible génération d'harmoniques (BROSSARD et al., 2009). L'étude se concentre alors sur la dynamique tourbillonnaire. Pour décrire la cinématique des tourbillons, une étude 2D a, dans un premier temps, été menée. L'utilisation de la PIV synchronisée avec la houle permet de déterminer des champs de vitesse et de vorticité et de suivre l'évolution spatio-temporelle des tourbillons.

L'ensemencement du canal est réalisé par électrolyse. Celle-ci permet de générer des microbulles d'hydrogène qui serviront de traceurs pour la PIV. La caméra utilisée dans cette expérience est constituée d'un capteur CCD de $1600 \times 1200$ pixels pouvant atteindre la fréquence maximale d'acquisition de $30 \mathrm{~Hz}$. Le laser pulsé (YAG) à deux têtes dispose d'une puissance de $120 \mathrm{~mJ}$ et d'une fréquence maximum de $15 \mathrm{~Hz}$. La caméra et le laser sont synchronisés sur la houle à l'aide d'un boîtier de synchronisation qui reçoit son signal d'entrée au moyen d'un codeur qui détecte chaque aller-retour du batteur. Pour cette expérience la caméra et le laser sont réglés pour obtenir dix paires d'images par période. 


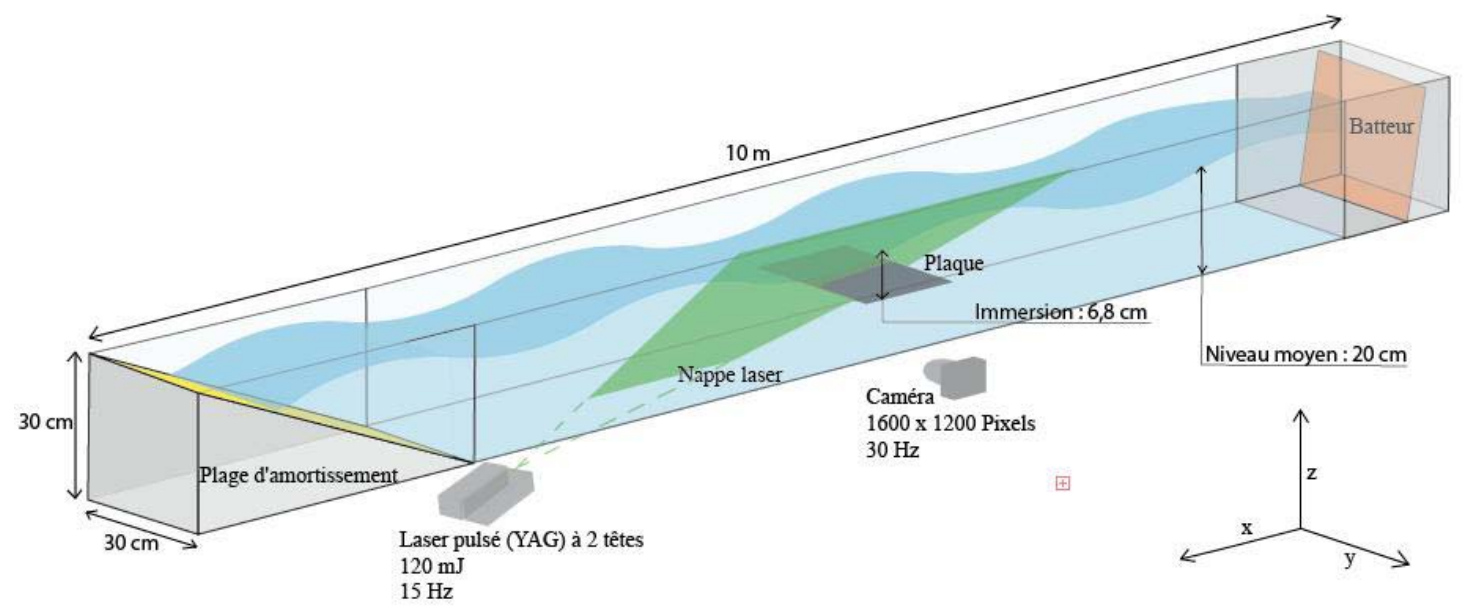

Figure 2. Configuration expérimentale.

\section{Résultats expérimentaux}

La PIV permet d'obtenir une évolution temporelle des champs de vitesse et de vorticité. L'évolution sur une période des champs de vorticité, moyennés par phase sur 50 périodes, est présentée figure 3. Les différentes grandeurs sont adimensionnées de la façon suivante : les échelles de longueur sont adimensionnées par h la hauteur d'eau ; les vitesses sont adimensionnées par U l'amplitude de la composante horizontale de vitesse à la surface libre pour une hauteur d'eau égale à l'immersion de la plaque; et la vorticité est adimensionnée par $U / \delta$ où $\delta$ est l'épaisseur de la couche limite dans le cas d'un écoulement oscillant :

$\delta=\sqrt{v T}$ avec $v$ la viscosité cinématique et T la période de la houle.

Le choix des échelles de vitesses et de vorticité est motivé par le fait que les vitesses au dessus de la plaque déterminent le développement de la couche limite sur la plaque et la formation des tourbillons. De plus, ces échelles permettent d'obtenir des champs de vitesse et de vorticité non distordus.

Sur une demi-période un tourbillon et une nappe de vorticité de signe opposé sont formés en bout de plaque et se détachent. La nappe de vorticité se déstabilise et forme un tourbillon contrarotatif. Le dipôle ainsi formé est advecté vers le fond par autoinduction pendant la seconde demi-période alors qu'un nouveau tourbillon se forme en bout de plaque. Ce dipôle se déstabilise au bout d'une période et demi environ.

Pour localiser les tourbillons à partir de ces champs de vitesse et de vorticité il existe plusieurs méthodes (HUNT et al., 1988; CHONG \& PERRY, 1990; JEONG \& HUSSAIN, 1994 ; GRAFTIEAUX et al., 2001). La méthode la plus efficace dans notre étude est celle de Jeong et Hussain dénommée " $\lambda 2$ factor". En effet, elle permet de localiser les minima de pression associés aux centres des tourbillons en cherchant les valeurs propres négatives de la somme des parties symétrique et antisymétrique du 


\section{XI ${ }^{\text {èmes }}$ Journées Nationales Génie Côtier - Génie Civil \\ Les Sables d'Olonne, 22-25 juin 2010}

tenseur gradient de vitesse toutes deux au carré. Cette méthode permet, entre autre, de distinguer un tourbillon dans une nappe de vorticité ce qui n'est pas possible en utilisant une méthode basée sur les maxima de vorticité comme critère de localisation.

La trajectoire moyenne des tourbillons calculée par la méthode du " $\lambda 2$ factor" est représentée figure 4.

L'évolution des tourbillons présente plusieurs phases qui se répètent à chaque période jusqu'à leur destruction au voisinage du fond du canal : une phase de stagnation où les tourbillons ne bougent quasiment pas et une phase d'advection. Ces deux phases correspondent respectivement à un creux de vague et à une crête de vague. Lorsque les tourbillons se rapprochent du fond du canal ils se déstabilisent et éclatent.

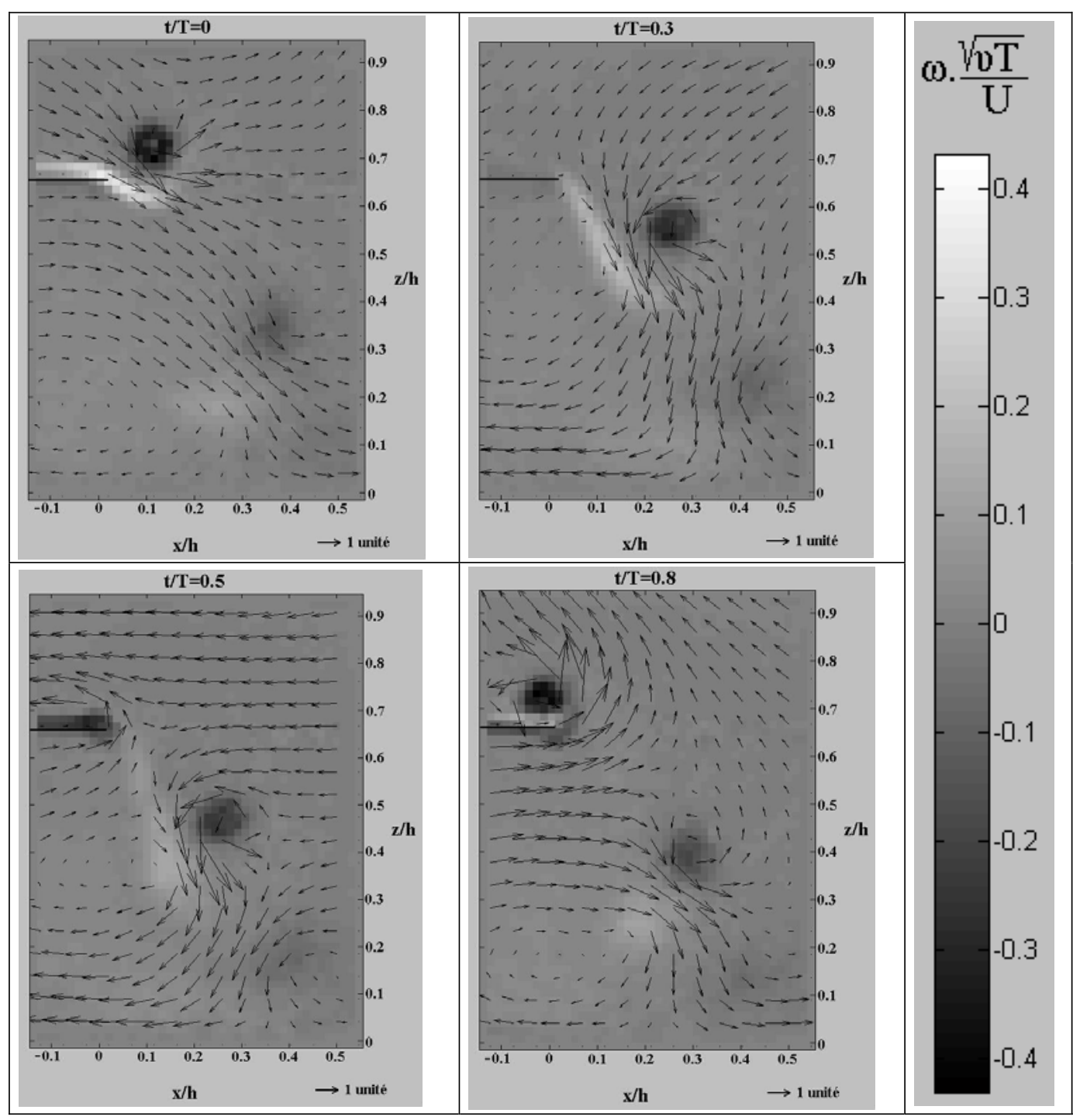

Figure 3. Champs de vorticité moyennés pour $t / T=0, t / T=0.3, t / T=0.5$ et $t / T=0.8$. 


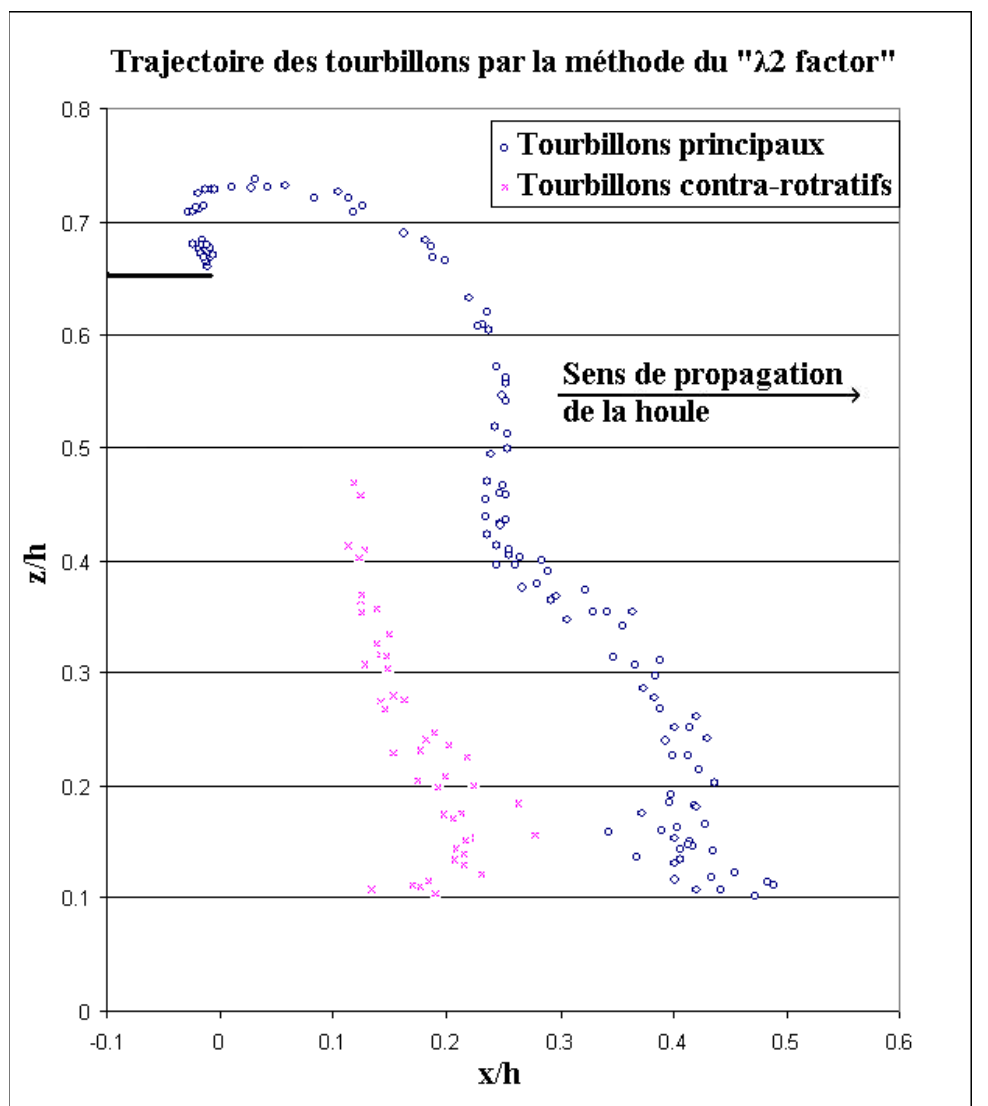

Figure 4. Trajectoire des tourbillons par la méthode du "ג2 factor".

Une étude des champs de divergence à permis de révéler l'existence d'un gradient de vitesse non nul suivant l'axe des tourbillons qui peut être associé aux déformations 3D de ceux-ci lors de leur évolution. L'observation des champs moyens de vorticité révèlent également que les maxima de vorticité ne sont pas toujours au centre des "patchs" de vorticité et que la distribution de vorticité a une forme annulaire. Une étude est en cours pour confirmer l'apparition de ce type de structures.

\section{Modèle numérique}

Le modèle numérique est très largement inspiré des travaux de BOULIER (1994, 1996 et 1997) qui ont depuis été repris en partie par MORDANE et al. (2008). Dans un premier temps, nous souhaitons reprendre exactement la même configuration numérique que celle de BOULIER (1996 et 1997) afin de pouvoir comparer les résultats obtenus de manière plus quantitatives avec ceux expérimentaux issus des nouvelles technologies expérimentales, la PIV notamment. Une fois les validations des résultats numériques ayant été réalisées grâce aux expériences, nous souhaitons obtenir des informations supplémentaires sur l'activité tourbillonnaire en utilisant d'autres paramètres, le modèle numérique pouvant balayer rapidement une plus large gamme de paramètres que l'expérience. Puis dans un second temps, nous espérons améliorer le modèle numérique 
initial de BOULIER (1996 et 1997) afin d'implémenter la prise en compte d'une houle non linéaire par exemple, suite à la présence de la plaque notamment.

Pour cette modélisation, on considère une plaque plane immergée dans une houle monochromatique. La plaque est horizontale (parallèle au fond du canal) et est supposée infiniment mince. Le fluide est supposé parfait, homogène, incompressible et non visqueux. Suite à la décomposition de Helmoltz du champ de vitesse $\vec{U}$ (équation 1), tout vecteur se décompose sous la forme d'une partie potentielle $\left(\overrightarrow{U_{h}}\right.$ vitesse due à la houle et $\overrightarrow{U_{p}}$ vitesse due à la présence de la plaque) et d'une partie rotationnelle $\left(\overrightarrow{U_{\omega}}\right.$ vitesse induite par le sillage tourbillonnaire) et on obtient :

$\vec{U}=\overrightarrow{U_{h}}+\overrightarrow{U_{p}}+\overrightarrow{U_{\omega}}$

$\overrightarrow{U_{h}}(\vec{x}, t)$, le champ de vitesse induit par la houle incidente.

Le champ de vitesse induit par la houle s'exprime à partir du modèle de Stokes en profondeur finie :

$$
\begin{gathered}
U_{h x}(\vec{x}, t)=\frac{\pi H}{T} \frac{\operatorname{ch}\left(2 \pi \frac{z}{\lambda}\right)}{\operatorname{sh}\left(2 \pi \frac{h}{\lambda}\right)} \cos \left(2 \pi\left(\frac{t}{T}-\frac{x}{\lambda}\right)\right) \\
U_{h z}(\vec{x}, t)=-\frac{\pi H}{T} \frac{\operatorname{sh}\left(2 \pi \frac{z}{\lambda}\right)}{\operatorname{sh}\left(2 \pi \frac{h}{\lambda}\right)} \sin \left(2 \pi\left(\frac{t}{T}-\frac{x}{\lambda}\right)\right)
\end{gathered}
$$

avec $\mathrm{H}$, l'amplitude de la houle; T, la période de la houle; $\mathrm{h}$, la hauteur d'eau au repos et $\lambda$, la longueur d'onde de la houle.

$\overrightarrow{U_{p}}(\vec{x}, t)$, le champ de vitesse induit par la plaque.

$\overrightarrow{U_{p}}(\vec{x}, t)$ est le champ de vitesse de l'écoulement qui permet de vérifier les conditions aux limites sur la plaque c'est à dire vérifier que la composante normale de la vitesse $\vec{U}$ à la plaque est nulle $(\vec{U} \cdot \vec{n}=0)$. Pour cela, la plaque est discrétisée en $\mathrm{N}$ facettes. Une équivalence doublet-tourbillon permet d'associer un filament rotationnel à chaque extrémité de facette. L'intensité de chaque filament est déterminée en considérant d'une part, la condition aux limites est vérifiée en chaque centre de facette et d'autre part, que la somme de la circulation des particules émise et celle due à la plaque reste nulle (condition de Kelvin). Pour plus de détail sur la méthode, le lecteur pourra se référer à BOULIER (1996), NITSCHE (1992) et BRATEC (2003).

$\overrightarrow{U_{\omega}}(\vec{x}, t)$, le champ de vitesse induit par le sillage tourbillonnaire.

En considérant le rotationnel de l'équation de Navier-Stokes nous obtenons l'équation de transport de vorticité en écoulement bidimensionnel :

$$
\frac{\partial \omega}{\partial t}+(\vec{U} \cdot \vec{\nabla}) \omega=v \Delta \omega
$$


avec $\omega=(\vec{\nabla} \times \vec{U}) \cdot \overrightarrow{e_{y}}$ et $v$ la viscosité cinématique. Si nous prenons le cas des configurations expérimentales présentées dans ce papier, le nombre de Reynolds basé sur la vitesse maximum au niveau de la plaque, la corde de celle-ci et la viscosité cinématique du fluide, nous donne un ordre de grandeur de l'ordre du millier. Nous pouvons en déduire qu'au premier ordre, les phénomènes visqueux ne seront pas dominants. Donc, en supposant que le fluide est non visqueux, on obtient :

$$
\frac{\partial \omega}{\partial t}+(\vec{U} \cdot \vec{\nabla}) \omega=0 \Leftrightarrow \frac{d \omega}{d t}=0
$$

L'écoulement étant supposé incompressible, la formule de Biot et Savart permet de calculer la vitesse $\overrightarrow{U_{\omega}}(\vec{x}, t)$ en tout point $\mathrm{M}$ du domaine $\mathrm{S}$ :

$$
\overrightarrow{U_{\omega}}(\vec{x}, t)=\int_{S} \vec{K}\left(\vec{x}-\vec{x}^{\prime}\right) \times \omega\left(\vec{x}^{\prime}\right) \overrightarrow{e_{y}} d S
$$

où $\vec{K}$ est le noyau de l'équation de Biot et Savart.

La forme discrète de cette équation peut alors s'écrire comme la somme des contributions d'un ensemble fini de filaments tourbillonnaires :

$$
\overrightarrow{U_{\omega}}(\vec{x}, t)=\sum_{j} \vec{K}\left(\vec{x}-\overrightarrow{x_{j}}\right) \times \Gamma_{j} \overrightarrow{e_{y}}
$$

où $\Gamma_{j} \approx \Gamma\left(\overrightarrow{x_{j}}\right) \approx \omega\left(\overrightarrow{x_{j}}\right) d S_{j}$ est la circulation associée au filament de la particule en $\overrightarrow{x_{j}}$ et qui a été émis à une extrémité de la plaque puis advectée par l'écoulement. L'expression du noyau de l'équation (6) montre une singularité lorsque deux particules sont proches l'une de l'autre ; le champ de vitesse $\overrightarrow{U_{\omega}}$ est alors régularisé (BRATEC, 2003).

Le fond du canal est modélisé par effet miroir. A chaque pas de temps, à chaque particule de l'écoulement est associée une particule symétrique par rapport au fond; de même la plaque plane elle aussi est symétrisée. Le même principe a été mis en œuvre pour la surface libre ; celle-ci ayant été linéarisée autour de sa position au repos.

La figure 5 présente le champ de vorticité instantané pour une simulation de plaque plane dans la houle avec les mêmes paramètres que ceux utilisés par BOULIER (1996) p. 133-137. De manière qualitative, les résultats obtenus sont très similaires à ceux qui avaient été obtenus lors de la précédente étude. On retrouve notamment, et de manière très claire, la présence de cette nappe de vorticité en forme de haricot (Cf. BOULIER (1996), figure 8.5 - p.137). Des comparaisons quantitatives en termes de trajectoires et intensité de tourbillons sont en cours de réalisation.

\section{Conclusion et remerciements}

L'étude expérimentale permet de mieux appréhender les tourbillons issus de la plaque immergée. Elle a révélé une déstabilisation tridimensionnelle des tourbillons. Une nouvelle expérience utilisant la stéréo-vidéo a déjà été lancée pour identifier les différents modes de perturbation des tourbillons.

Actuellement le modèle numérique est encore en cours d'implémentation. Les comparaisons des résultats issus de la configuration numérique de BOULIER (1996 et 
1997) avec les résultats obtenus grâce aux nouvelles méthodes expérimentales (PIV) seront réalisées et feront l'objet d'une prochaine communication. Nous pourrons alors évaluer et améliorer, si nécessaire, la prise en compte de la diffusion ou la modification du champ de houle initial suite à la présence de la plaque, etc.

Cette étude, en partie financée par la Région Haute Normandie, montre toute la complexité de l'écoulement à proximité des plaques immergées. Son approfondissement permettra de mieux appréhender ces écoulements.

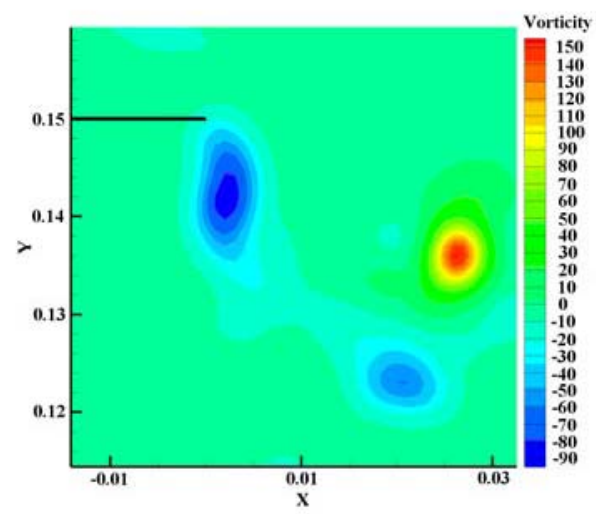

Figure 5. Visualisation du champ tourbillonnaire instantané pour une simulation de plaque plane dans la houle avec les mêmes paramètres que ceux utilisés par BOULIER (1996) p. 133-137.

\section{Références Bibliographiques}

BOULIER B. (1996). Evolution spatio-temporelle de structures tourbillonnaires dans la houle. Thèse de doctorat, Université du Havre, France.

BOULIER B., HEMON A., RIVOALEN E., BROSSARD J. (1997). Etude expérimentale et numérique de l'écoulement au voisinage d'une plaque immergée dans la houle. $6^{\text {ème }}$ Journées hydrodynamique, Nantes.

BRATEC H. (2003). Modélisation numérique des jets : application aux inverseurs de poussée. Thèse de doctorat, Université du Havre, France.

BROSSARD J., PERRET G., BLONCE L., DIEDHOU A. (2009). Higher harmonics induced by a submerged horizontal plate and a submerged rectangular step in a wave flume. Coastal engineering, vol. 56, $\mathrm{n}^{\circ}$ 1, pp 11-22. doi:10.1016j.coastaleng.2008.06.002

CARTER (2005). Wave energy converters and a submerged horizontal plate. Thesis of University of Hawaii, Ocean and Resources Engineering, 251 p.

CHANG K.-A, HSU T.-J., LIU P. L.-F. (2001). Vortex generation and evolution in water waves propagating over a submerged rectangular obstacle: Part I. Solitary waves. Coastal Engineering 44, pp 13-36. doi:10.1016/S0378-3839(01)00019-9

CHANG K.-A, HSU T.-J., LIU P. L.-F. (2005). Vortex generation and evolution in water waves propagating over a submerged rectangular obstacle: Part II. Cnoidal waves. Coastal Engineering 52, pp 257-283. doi:10.1016j.coastaleng.2004.11.006 
CHERFILS J.-M., BLONCE L., PINON G., RIVOALEN E. (2009). Modélisation de l'interaction houle-plaque horizontale immergée par la méthode SPH. $19^{\text {ème }}$ Congrès Français de Mécanique Marseille, 24-28 août.

CHONG M. S., PERRY A.E. (1990) A general classification of three-dimensional flow fields. Phys. Fluids A2(5), pp 765-777.

GRAFTIEAUX L., MICHARD M., GROSJEAN N. (2001). Combining PIV, POD and vortex identification algorithms for the study of unsteady turbulent swirling flows. Meas. Sci. Technol. 12 (2001), pp 1422-1429. doi:10.1088/0957-0233/12/9/307

GRAW K.-U. (1993). The submerged plate wave energy converter - A new type of wave energy device. ODEC, Mumoran, Hokkaido, Japan, pp 1-4.

HUANG C.-J., DONG C.-M. (1999). Wave deformation and vortex generation in water waves propagating over a submerged dike. Coastal Engineering 37, pp 123-148. doi:10.1016/S0378-3839(99)00017-4

HUNT J., WRAY A., MOIN P. (1988). Eddies, stream, and convergences zones in turbulent flows. Center for turbulent Research Report, CTR-S88, pp 193-208.

JEONG J., HUSSAIN F. (1994). On the identification of a Vortex. J. of Fluid Mech., vol. 285, pp 69-94. doi:10.1017/S0022112095000462

MASSEL S.-R. (1983). Harmonic generation by waves propagating over a submerged plate. Coastal Engineering 7, pp 357-380. doi:10.1016/0378-3839(83)90004-2

MORDANE S., MANGOUD G., CHAGDALI M. (2008). Etude numérique de l'interaction houle-plaque immergée. Xèmes Journées Nationales Génie Côtier - Génie Civil, 14-16 octobre 2008, pp 405-414. doi:10.5150/jngcgc.2008.039-M

NITSCHE M. (1992). Axisymmetric vortex sheet roll-up. PhD thesis, University of Michigan.

PATARAPANICH M. (1984). Maximum and zero reflection from submerged plate. J. of Waterway Port Coastal and Ocean Eng., ASCE, 110(2), pp 171-181. doi:10.1061/(ASCE)0733-950X(1984)110:2(171)

PATARAPANICH M., CHEONG H.-F. (1989). Reflexion and transmission characteristics of a regular and random wave from a submerged horizontal plate. Coastal Eng., 13(2), pp 161-182. doi:10.1016/0378-3839(89)90022-7

SUMER B. M., WHITEHOUSE R. J. S., TORUM A. (2001). Scour around coastal structures: a summary of recent research. Coastal Engineering 44, pp 153-190. doi:10.1016/S0378-3839(01)00024-2

TING F. C.K., KIM Y.-K. (1994). Vortex generation in water waves propagation over a submerged obstacle, Coastal Engineering 24, pp 23-49. doi:10.1016/0378-3839(94)90025-6 